\title{
THERMAL ANALYSIS AND OPTIMIZATION OF A HEAT REGENERATOR COMPOSED BY TWO COUPLED MOVING BED HEAT EXCHANGERS
}

\author{
J.A. Almendros-Ibáñez ${ }^{\S}$, A. Soria-Verdugo, U. Ruiz-Rivas and D. Santana \\ Carlos III University of Madrid, ISE Research Group, \\ Thermal and Fluid Engineering Department, \\ Avda. de la Universidad 30, 28911 Leganés, Madrid, Spain \\ ${ }^{\S}$ Correspondence author. Tel.-Fax: +34916248884 - 9430; Email: jalmendr@ing.uc3m.es
}

\begin{abstract}
This work presents a study to optimize the performance of a heat regenerator composed by two coupled moving bed heat exchangers (MBHE). A MBHE is used to recover heat, from a hot gas stream, and the other one is used to preheat an air stream. A direct application might be a gasifier. The heat exchangers performance was studied in two cases, considering or not the conduction heat transfer in the solid phase. When the solid conduction is taken into account, a numerical solution is obtained, while an analytical solution is possible when the conduction terms are neglected. In both cases, the optimum values of bed length (in the air flow direction) and particle diameter were obtained from an exergy point of view. Finally, an energy optimization of the heat regenerator was carried out, obtaining the optimal heat regenerator dimensions as a function of gas velocity and gas flow rate.
\end{abstract}

\section{INTRODUCTION}

One of the main problems in the performance of a biomass or coal gasification plant is the cleanup and filtration of the gas yielded in the gasification process before it is introduced in a power device (e.g. a gas turbine or an internal combustion engine). Different equipments have been proposed for hot gas particulate removal, such as electrostatic precipitators, ceramic filters, scrubbers, bag filters and granular filters. The ceramic and bag filters are the most popular. However, the collected particles can clog the fileter and the pressure drop increases [Smid et al., 2005a]. The same difficulty is found in fixed granular beds [Socorro et al., 2006]. Different means have been developed to clean the surface of these filters. For example, to have a dual system, where one filter is working while the other one is being regenerated, or to provide a short pulse of pressure in the direction reverse to the normal flow. The latter has to be applied carefully in ceramic filters, because the pulses and vibrations can result in a reduction of its life and can ever result in the breakage of the ceramic filter [Hsiau et al., 2004].

On the other hand, moving bed filters are increasing in interest as key component in integrated gasification combined cycle as well as in pressurized fluidized bed combustors. Smid et al. [2005b] made a complete review of the patent literature about moving bed filters and their equipment in different countries all around the world. Moving beds do not become clogged and can filter the gas at high temperatures, whereas ceramic filters have several problems to work at temperatures over 


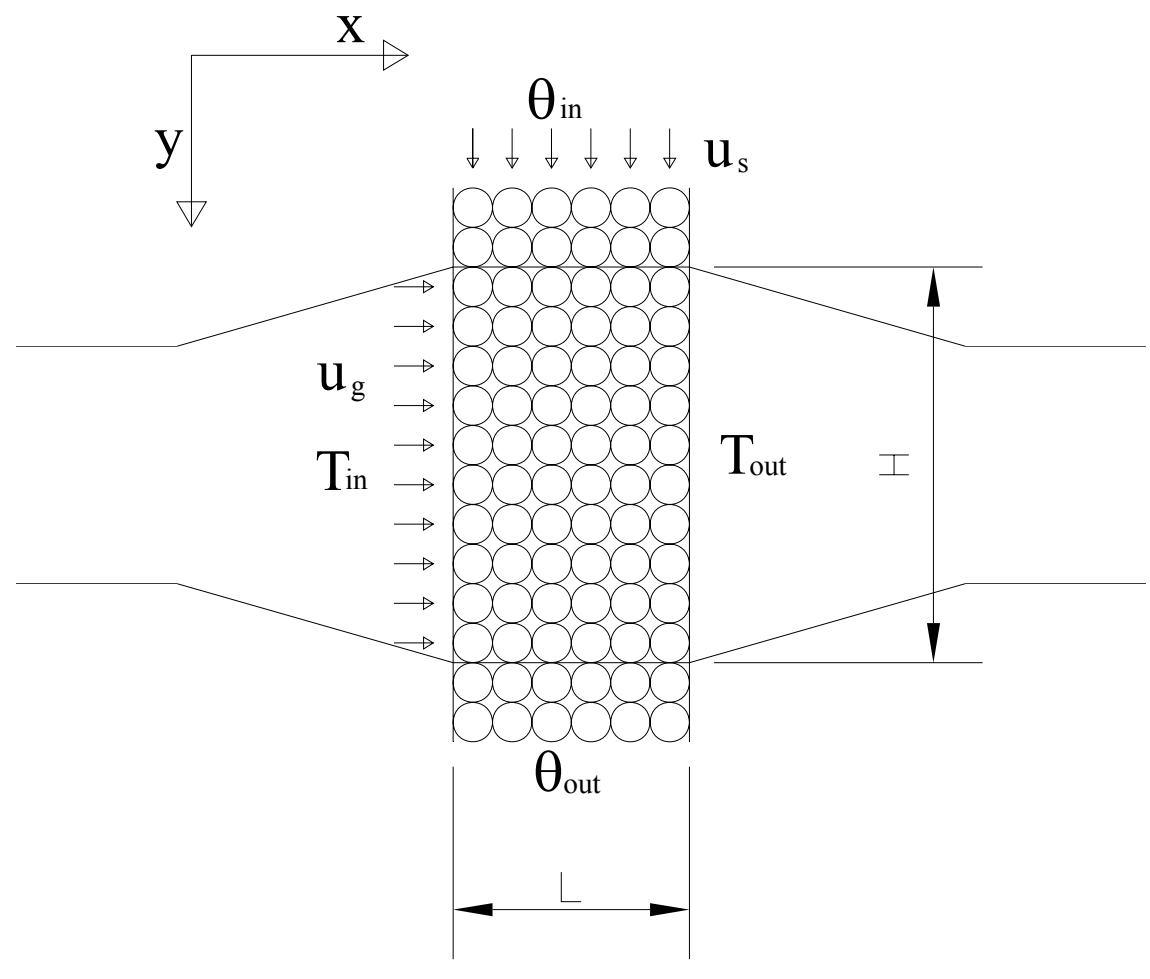

Figure 1.Schematic of a Moving Bed Heat Exchanger

$400^{\circ} \mathrm{C}$ [Longanbach, 1998]. Several studies can be found in the literature about flow patterns and particles velocity in moving beds, as for example the works by Hsiau et al. [1999, 2001, 2005] as well as on the heat transfer between gas and particles in fixed or moving beds [Yagi and Kunii, 1960; Yagi et al., 1960; Macías-Machín et al., 1991; Achenbach, 1995; Henriquez and MaciasMachín, 1997; Socorro et al., 2006]. In contrast, there is not so much information about the optimization of the moving bed dimensions from an energy or exergy point of view [Soria-Verdugo et al., 2007].

The focus of this article is on the optimization of the dimensions of a heat regenerator, composed by two moving bed heat echangers (MBHE hereafter). For such purpose, in the first part of the work a thermal analysis and an exergy optimization [Bejan, 1996] of the dimensions of a MBHE are performed. The last part of this work devotes to the optimization of the whole heat regenerator, composed by two coupled MBHE (one to recover heat from hot gases and one to preheat ambient air). This is done by means of an energy optimization.

The experimental conditions of Henriquez and Macías-Machín [1997] have been considered as the input data for the nominal case. The gas is air and its superficial gas velocity is $0.9 \mathrm{~m} / \mathrm{s}$. The solids are $1 \mathrm{~mm}$ diameter spheres of steel moving down with a velocity of $5 \mathrm{~cm} / \mathrm{min}$. The MBHE is $\mathrm{L}=15$ $\mathrm{cm}$ length (in the direction of the gas flow) and $\mathrm{H}=50 \mathrm{~cm}$ height (in the direction of the particle flow). A homogenous void fraction $\varepsilon=0.4$ is assumed.

\section{GOVERNING EQUATIONS}

Figure 1 shows a schematic of a MBHE. A flow of particles moving down with a velocity $u_{s}$ interchanges heat with a gas stream moving in cross flow with a velocity $u_{g}$. The heat 
regenerator will consist of two coupled MBHE like the one showed in figure 1, where the outlet of particles of the MBHE that recovers heat is the inlet of the one used as a preheater.

The general 2-phase equations governing heat transfer for the MBHE showed in figure 1 are given by the equation system (1)

$$
\begin{aligned}
& \varepsilon \rho_{g} c_{p g}\left(\frac{\partial \bar{T}}{\partial t}+\mathbf{u}_{\mathrm{g}} \cdot \nabla \bar{T}\right)=\nabla \cdot\left(k_{g} \nabla \bar{T}\right)+h_{s} a_{s}(\bar{\theta}-\bar{T})-U_{g}\left(\bar{T}-\bar{T}_{a m b}\right)-\dot{q}_{r a d} \\
& (1-\varepsilon) \rho_{s} C_{s}\left(\frac{\partial \bar{\theta}}{\partial t}+\mathbf{u}_{\mathrm{s}} \cdot \nabla \bar{\theta}\right)=\nabla \cdot\left(k_{s} \nabla \bar{\theta}\right)+h_{s} a_{s}(\bar{T}-\bar{\theta})-U_{s}\left(\bar{\theta}-\bar{\theta}_{a m b}\right)+\dot{q}_{r a d}
\end{aligned}
$$

where $\bar{T}$ and $\bar{\theta}$ are the gas and solid temperature respectively and $a_{s}$ is the superficial particle area per unit of bed volume. The last two terms of the right side of each equation represent the heat losses to the surroundings and the heat transfer by radiation between solid and gas respectively. In order to simplify the problem, the system is assumed to be well isolated, i.e. the global heat transfer coefficients $U_{g}$ and $U_{s}$ are low and these terms can be neglected. Moreover, the maximum temperature in the experimental conditions of Henriquez and Macías-Machín [1997], which are used in this work, does not excess $100{ }^{\circ} \mathrm{C}$. Thus, radiation heat transfer $q_{\text {rad }}$ is also neglected. With these assumptions, the governing equations reduce to

$$
\begin{aligned}
& \varepsilon \rho_{g} c_{p g}\left(\frac{\partial \bar{T}}{\partial t}+\mathbf{u}_{\mathrm{g}} \cdot \nabla \bar{T}\right)=\nabla \cdot\left(k_{g} \nabla \bar{T}\right)+h_{s} a_{s}(\bar{\theta}-\bar{T}) \\
& (1-\varepsilon) \rho_{s} C_{s}\left(\frac{\partial \bar{\theta}}{\partial t}+\mathbf{u}_{\mathrm{s}} \cdot \nabla \bar{\theta}\right)=\nabla \cdot\left(k_{s} \nabla \bar{\theta}\right)+h_{s} a_{s}(\bar{T}-\bar{\theta})
\end{aligned}
$$

The equation system (2) can be further simplified comparing the gas conductivity with the solid conductivity:

$$
\frac{k_{g}}{k_{s}}=\frac{0.03 \mathrm{~W} / \mathrm{m} \cdot \mathrm{K}}{15^{\mathrm{W}} / \mathrm{m} \cdot \mathrm{K}}=2 \times 10^{-3} \ll 1
$$

The gas conductivity is several orders of magnitude lower than the solid conductivity and can be neglected from equation (2). With this additional simplification and assuming a 2-D geometry and steady state, the general governing equations result in:

$$
\begin{aligned}
& \varepsilon \rho_{g} c_{p g}\left(u_{g} \cdot \frac{\partial \bar{T}}{\partial x}\right)=h_{s} a_{s}(\bar{\theta}-\bar{T}) \\
& (1-\varepsilon) \rho_{s} C_{s}\left(u_{s} \cdot \frac{\partial \bar{\theta}}{\partial y}\right)=k_{s x} \frac{\partial^{2} \bar{\theta}}{\partial x^{2}}+k_{s y} \frac{\partial^{2} \bar{\theta}}{\partial y^{2}}+h_{s} a_{s}(\bar{T}-\bar{\theta})
\end{aligned}
$$

The equation system (4) can be written in compact form as follows

$$
(1-\varepsilon) \rho_{s} C_{s}\left(u_{s} \cdot \frac{\partial \bar{\theta}}{\partial y}\right)-k_{s x} \frac{\partial^{2} \bar{\theta}}{\partial x^{2}}-k_{s y} \frac{\partial^{2} \bar{\theta}}{\partial y^{2}}=h_{s} a_{s}(\bar{T}-\bar{\theta})=-\varepsilon \rho_{g} c_{p g}\left(u_{g} \cdot \frac{\partial \bar{T}}{\partial x}\right)
$$


and in a non-dimensional form

$$
\frac{\partial \theta}{\partial \eta}-K_{x} \frac{\partial^{2} \theta}{\partial \xi^{2}}-K_{y} \frac{\partial^{2} \theta}{\partial \eta^{2}}=T-\theta=-\frac{\partial T}{\partial \xi}
$$

where

$$
\begin{gathered}
\theta=\frac{\bar{\theta}-\bar{\theta}_{i n}}{\bar{T}_{i n}-\bar{\theta}_{i n}}, \quad T=\frac{\bar{T}-\bar{\theta}_{i n}}{\bar{T}_{i n}-\bar{\theta}_{i n}} \\
\xi=\frac{x h_{p} a_{p}}{\varepsilon \rho_{g} u_{g} c_{p g}}, \quad \eta=\frac{y h_{p} a_{p}}{(1-\varepsilon) \rho_{s} u_{s} C_{s}} \\
K_{\xi}=\frac{h_{s} a_{s} k_{s x}}{\left(\varepsilon \rho_{g} u_{g} c_{p g}\right)^{2}}, \quad K_{\eta}=\frac{h_{s} a_{s} k_{s y}}{\left((1-\varepsilon) \rho_{s} u_{s} C_{s}\right)^{2}}
\end{gathered}
$$

The gas and particle temperatures have been non-dimensionalized according to equation ( 7 ). The non-dimensional temperatures vary between 0 and 1 . The variables $\xi$ and $\eta$ are the nondimensional horizontal and vertical coordinatesn respectively. $K_{\xi}$ and $K_{\eta}$ are the non-dimensional conductivities in the direction of the gas flow and in the direction of the particle flow respectively.

The value of the thermal conductivity in the direction of the gas flow $k_{s x}$ was obtained using a correlation proposed by Krupiczka [1967] and the thermal conductivity in the direction of the particle flow $k_{s y}$ using the equation suggested by Yagi et al. [1960]. These expressions have been used by Marb and Vortmeyer [1988] in their study of a moving bed reactor. The convection heat transfer coefficient $h_{s}$ was obtained with a correlation proposed by Achenbach [1995].

With the data of Henriquez and Macías-Machín [1997], the limit values of the non-dimensional coordinates are:

$$
\xi_{\max }=\xi(x=L) \approx \eta_{\max }=\eta(y=H) \approx 350
$$

resulting in a square heat exchanger when it is represented in the non-dimensional variables. When $\eta_{\max }=\xi_{\max }$, the heat transfer process is optimized, as shown in Soria-Verdugo et al. [2007].

Numerical Solution In order to solve the non-dimensional equation system (6) a set of boundary conditions is needed. Marb and Vortmeyer [1988] studied a similar problem for six different sets of boundary conditions and compared their numerical results with experiments. They suggested that the boundary conditions showed in table 1, gave numerical results that fitted better to the experimental data. They argued that the boundary condition at $\eta=0$ (Danckwerts boundary condition) performs the continuity requirements for the energy flux, while other conditions do not perform it. Thus, the same boundary conditions have been employed in this work.

The differential equation system (6) was solved numerically using a finite difference technique. The elliptic character of the equations was transformed into parabolic adding a temporal derivate into the solid equation. The first derivate were discretized using an up-wind scheme and the second derivates using central differences, according to equations (11) and (12). 
Table 1

Boundary conditions

\begin{tabular}{|c|l|l|}
\hline \multirow{2}{*}{ Particle } & $\xi=0$ & $\frac{\partial^{2} \theta}{\partial \xi^{2}}=0$ \\
\cline { 2 - 3 } & $\xi=\xi_{\max }$ & $\frac{\partial^{2} \theta}{\partial \xi^{2}}=0$ \\
\cline { 2 - 3 } & $\eta=0$ & $\theta=K_{\eta} \cdot \frac{\partial \theta}{\partial \eta}$ \\
\cline { 2 - 3 } & $\eta=\eta_{\max }$ & $\frac{\partial \theta}{\partial \eta}=0$ \\
\hline Gas & $\xi=0$ & $T=1$ \\
\hline
\end{tabular}

$$
\begin{gathered}
\frac{\theta_{j, i}^{k+1}-\theta_{j, i}^{k}}{\Delta t}=\frac{\theta_{j, i}^{k}-\theta_{j-1, i}^{k}}{\Delta \eta}+K_{\xi} \frac{\theta_{j, i-1}^{k}-2 \cdot \theta_{j, i}^{k}+\theta_{j, i+1}^{k}}{(\Delta \xi)^{2}}+K_{\eta} \frac{\theta_{j-1, i}^{k}-2 \cdot \theta_{j, i}^{k}+\theta_{j+1, i}^{k}}{(\Delta \eta)^{2}}+\left(T_{j, i}^{k}-\theta_{j, i}^{k}\right) \\
\frac{T_{j, i}^{k+1}-T_{j, i-1}^{k+1}}{\Delta \xi}=\left(\theta_{j, i}^{k+1}-T_{j, i}^{k+1}\right)
\end{gathered}
$$

Combining equations (11) and (12), the non-dimensional solid temperature at time $\mathrm{k}+1$ can be obtained from the solution at time $\mathrm{k}$ as follows.

$$
\begin{aligned}
\theta_{j, i}^{k+1}= & \theta_{j-1, i}^{k} \cdot\left(\frac{\Delta t}{\Delta \eta}+\frac{\Delta t}{(\Delta \eta)^{2}} \cdot K_{\eta}\right)+\theta_{j+1, i}^{k} \cdot\left(\frac{\Delta t}{(\Delta \eta)^{2}} \cdot K_{\eta}\right)+ \\
& \theta_{j, i}^{k} \cdot\left(1-\frac{\Delta t}{\Delta \eta}-\frac{2 \cdot \Delta t}{(\Delta \eta)^{2}} \cdot K_{\eta}-\frac{2 \cdot \Delta t}{(\Delta \xi)^{2}} \cdot K_{\xi}-\Delta t\right)+ \\
& \theta_{j, i-1}^{k} \cdot\left(\frac{\Delta t}{(\Delta \xi)^{2}} \cdot K_{\xi}\right)+\theta_{j, i+1}^{k} \cdot\left(\frac{\Delta t}{(\Delta \xi)^{2}} \cdot K_{\xi}\right)+T_{j, i}^{k} \cdot \Delta t
\end{aligned}
$$

Then, the non-dimensional gas temperature was obtained from equation (14).

$$
T_{j, i}^{k+1}=\frac{\theta_{j, i}^{k+1} \cdot \Delta \xi+T_{j, i-1}^{k+1}}{1+\Delta \xi}
$$

The initial solution for time step $\mathrm{k}=0$ was the analytical solution obtained neglecting conduction heat transfer. The procedure to obtain it is showed in the next section.

Analytical Solution Neglecting Solid Conductivity The set of equations (6) has an analytical solution when the conduction terms are negligible, which is usually accepted for high Reynolds numbers [Vortmeyer and Schaefer, 1974]. Then, the following expression is obtained.

$$
\frac{\partial \theta}{\partial \eta}=(T-\theta)=-\frac{\partial T}{\partial \xi}
$$

The two boundary conditions needed to solve the equation system (15) are: 


$$
T(\xi=0)=1, \quad \theta(\eta=0)=0
$$

With such conditions, according to Saastamoinen [2003] and several other authors, the analytical solution for the non-dimensional gas and solid temperatures are

$$
\begin{gathered}
T=e^{-\xi-\eta} \sum_{j=0}^{\infty} \frac{\eta^{j}}{j !} \sum_{k=0}^{j} \frac{\xi^{k}}{k !} \\
\theta=1-e^{-\eta-\xi} \sum_{j=0}^{\infty} \frac{\xi^{j}}{j !} \sum_{k=0}^{j} \frac{\eta^{k}}{k !}
\end{gathered}
$$

\section{HEAT TRANSFER ANALYSIS OF A MBHE}

Figure 2 show respectively the non-dimensional gas and solid temperature profiles. Graphs (a) and (b) show the results obtained numerically, solving equation system (6) for the nominal case. The values of the conductivities in both directions were $K_{\xi}=4.15$ and $K_{\eta}=51.12$. Graphs (c) and (d) show the results obtained analytically, neglecting heat transfer due to conduction $\left(K_{\zeta}=K_{\eta}=0\right)$. In all cases the heat transfer occurs in the main diagonal of the MBHE. Temperature differencies
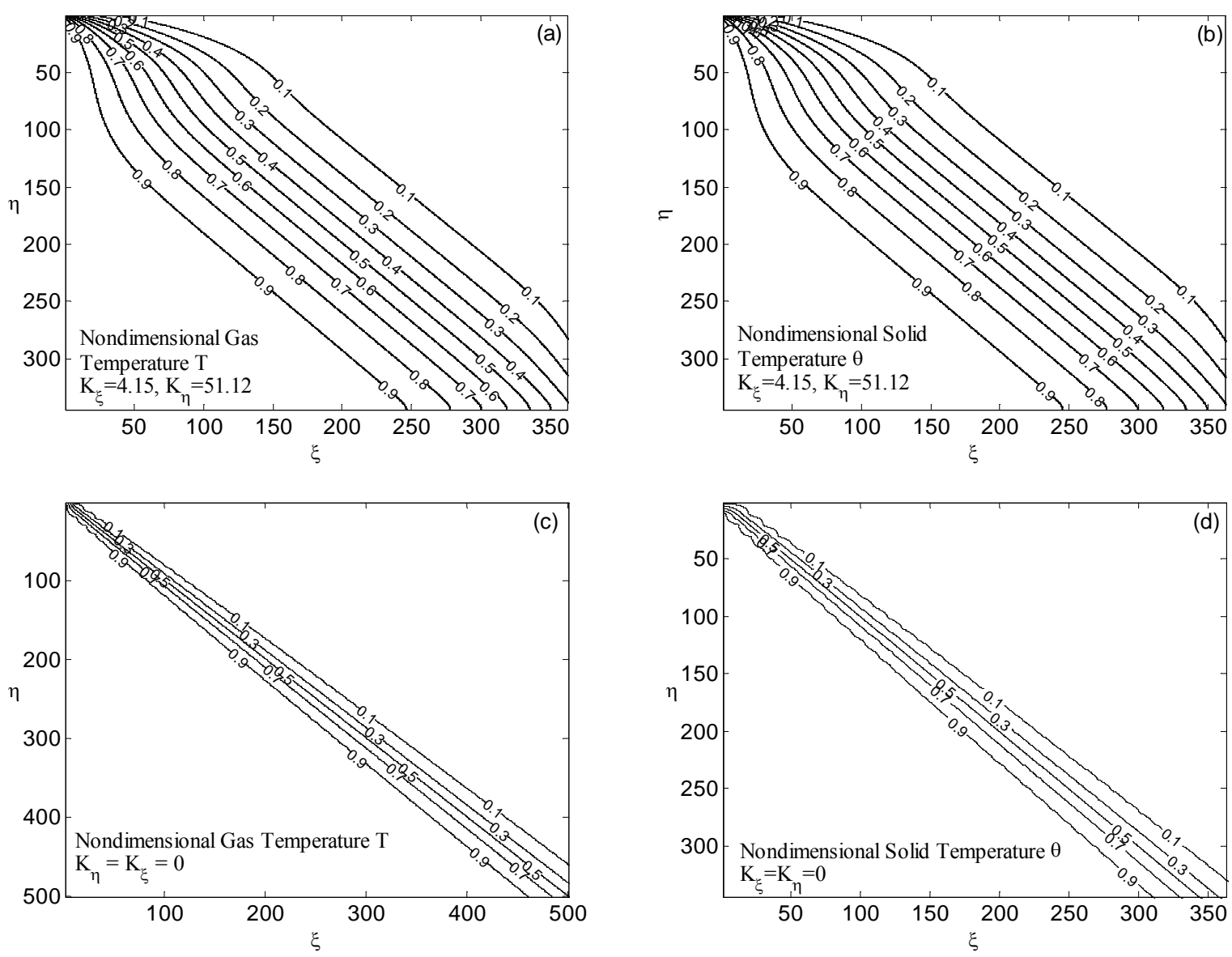

Figure 2. Non-dimensional gas and solid temperature profiles. (a) and (b): numerical results. (c) and (d) analytical results neglecting conduction effects. 
appear only in that zone. When conduction effects are neglected, the heat is only transferred by convection from the hot gas to the cold solids in a narrow region of the bed. In contrast, when conduction is taken into account, part of the heat is transferred by conduction in the solid phase due to differences in the solid phase temperature. As a result, the width of the region where $T$ and $\theta$ change increases notably when conduction is included.

The effect of the inlet boundary conditions is also appreciable in figure 2 in the region close to $\xi=\eta=0$. In graphs 2 (a) and (b) the solid conductivity was taken into account at $\eta=0$ (see table 1). Consequently the isothermal lines are displaced to $\eta=0$. In contrast, when conduction is neglected, the isotherm lines are symmetric with respect to the main diagonal $\xi=\eta$ of the moving bed.

Nevertheless, the mean outlet temperatures of gas and solids do not differ much whether conduction effects are neglected or not. These differences are less important for high values of $\xi_{\max }$ and $\eta_{\max }$, as it is the case. Consequently, the general inlet-outlet performance of a MBHE can be appropriately described using the analytical solution obtained from equations (17) and (18).

\section{EXERGY OPTIMIZATION OF A MBHE}

The MBHE studied in the previous section has also been analyzed and optimized from an exergy point of view. Different configurations of the MBHE, varying the particle size and the length of bed, were studied in order to find the optimal pair that minimizes the exergy destroyed during the steady state operation of the MBHE.

The exergy balance applied to the MBHE gives the exergy destruction as

$$
A_{d}=\overline{T_{A}}\left[\dot{m}_{g} c_{p g}\left(\operatorname{Ln}\left(\overline{\frac{T_{\text {out }}^{*}}{\bar{T}_{\text {in }}}}\right)-\frac{\gamma-1}{\gamma} \operatorname{Ln}\left(\frac{P_{\text {in }}-\Delta P}{P_{\text {in }}}\right)\right)+\dot{m}_{s} C_{s} \operatorname{Ln}\left(\overline{\frac{\theta_{\text {out }}^{*}}{\overline{\theta_{\text {in }}}}}\right)\right]
$$

where $\overline{T_{\text {out }}^{*}}$ and $\overline{\theta_{\text {out }}^{*}}$ are the mean gas and solid temperatures at the outlet sections of the MBHE, $\gamma$ is the specific heat ratio of air, $P_{i n}$ is the gas pressure at the inlet section and $\Delta P$ is the pressure drop of the gas crossing the moving bed. Exergy is destroyed by two mechanisms: heat transfer between the gas and the solids and pressure drop of the gas crossing the bed.

The gas pressure drop was calculated using Ergun equation (Ergun [1952]). Although Ergun equation was obtained for fixed (instead of a moving) beds, in our study the solid velocity is orders of magnitude lower than the gas velocity. Therefore, the particle movement can be neglected for pressure drop calculation.

Figure 3 shows the exergy destruction maps for the nominal case varying the length of the bed from 4 to $30 \mathrm{~cm}$ and the diameter of the particles between 1 and $10 \mathrm{~mm}$. Graph (a) shows the results obtained for the general case and grapgh (b) is obtained using the analytical solution (equations (17) and (18)) that neglects the effect of solid conductivity. The differences between both exergy maps are associated with small differences in the mean outlet temperatures. 


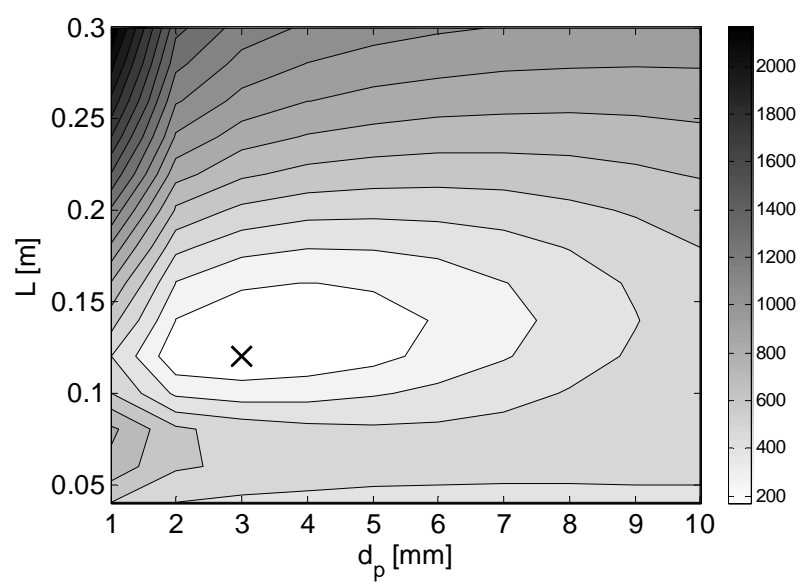

(a)

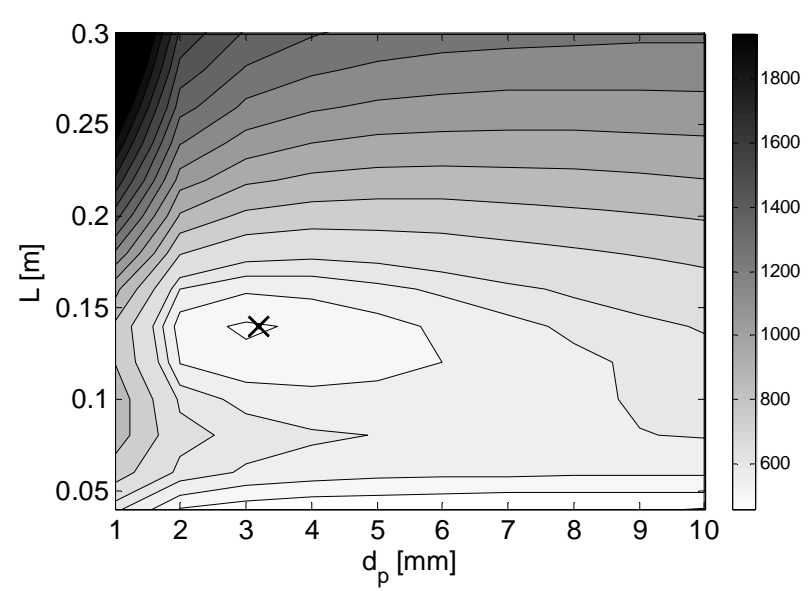

(b)

Figure 3. Destroyed exergy maps for the nominal case $\left(\mathrm{u}_{\mathrm{g}}=1.5 \mathrm{~m} / \mathrm{s}\right)$ varying the length of the bed and the particle diameter. (a) includes conduction effects and in (b) conduction effects are neglected. The crosses indicate the optimum point. Scale in watts

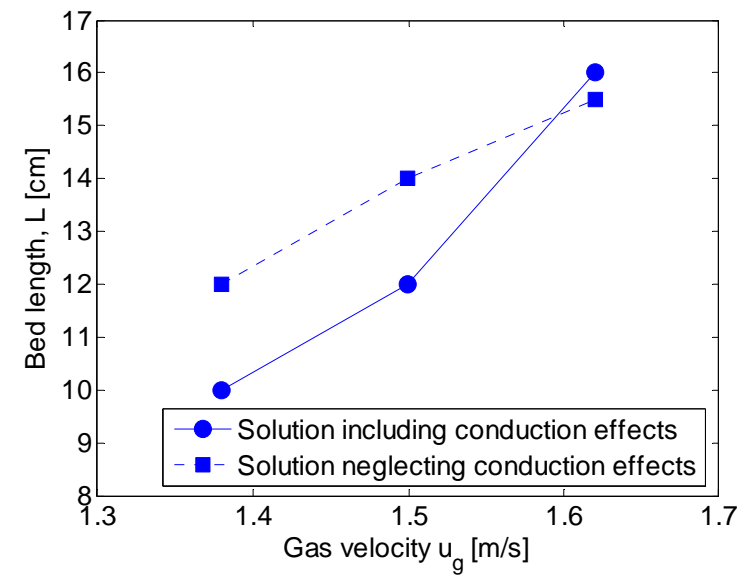

(a)

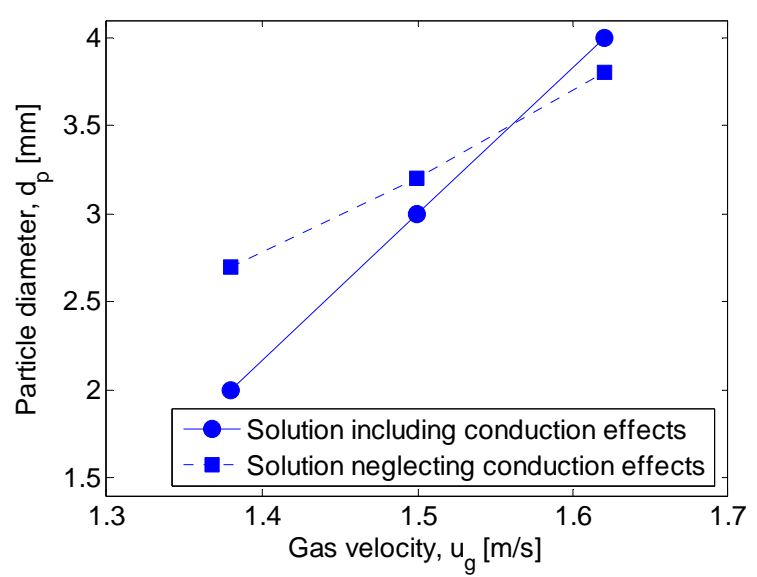

(b)

Figure 4. Evolution of the optimum particle diameter (a) and the optimum bed length (b) increasing the gas velocity including (solid line) or not (dashed line) conduction effects

When the size of the bed is small, the effect of the conductivity in the mean outlet temperatures is more important and thereby these differences are more noticeable for small bed lengths $(L \leq 0.1 \mathrm{~m})$. The crosses in figure 2 indicate the optimum point, showing good agreement in both cases.

Figure 4 shows the evolution of the optimum particle diameter and the optimum bed length with the superficial gas velocity. A solid line indicates the results obtained numerically (taken into account conduction heat transfer) and a dashed line the results obtained neglecting it. In both cases the same tendency is observed: the optimum particle diameter and the optimum bed length increase with the superficial gas velocity. Therefore, the analytical solution (equations (17) and (18)) can be used, as first approximation, to obtain the optimal performance of the MBHE. 


\section{ENERGY OPTIMIZATION OF THE HEAT REGENERATOR}

In order to study the global energy transfer parameters the complete regenerator configuration with two coupled MBHEs should be analysed. Figure 5 shows the configuration considered for calculations. The inlet and outlet dimensions (in the particle flow direction), and the gap between the two exchangers are designed to minimize possible leaks and shortcuts between the two flows. In this section, conduction heat transfer is neglected and thus the mean inlet and outlet gas temperatures were calculated analytically.

The main energetic parameters of the heat regenerator are the heat transferred between gas and

particles, $\dot{Q}$, and the power consumed to both raise the particles and pump the gas, $\dot{W}$. These parameters can be calculated per unit of gas mass flow for a square heat exchanger $(\xi(L)=\eta(H))$ using equation (20)

$$
\begin{aligned}
& \frac{\dot{Q}}{\dot{m}_{g}}=c_{p g} \cdot\left(\overline{T_{\text {in }}^{*}}-\overline{T_{\text {out }}^{*}}\right) \\
& \frac{\dot{W}}{\dot{m_{g}}}=\frac{1}{\eta_{s}} \cdot \frac{c_{p g}}{C_{s}} \cdot g \cdot(10 L+2 H)+\frac{1}{\eta_{g}} \cdot \frac{\Delta P}{\rho_{g}}
\end{aligned}
$$

Heat transfer and power consumption are shown in Figure 6 for a range of gas velocities and MBHE lengths. The nominal particle diameter and velocity, as well as efficiencies of 10\% and 50\% for raising the particles and pumping the gas respectively, have been used for the calculation.

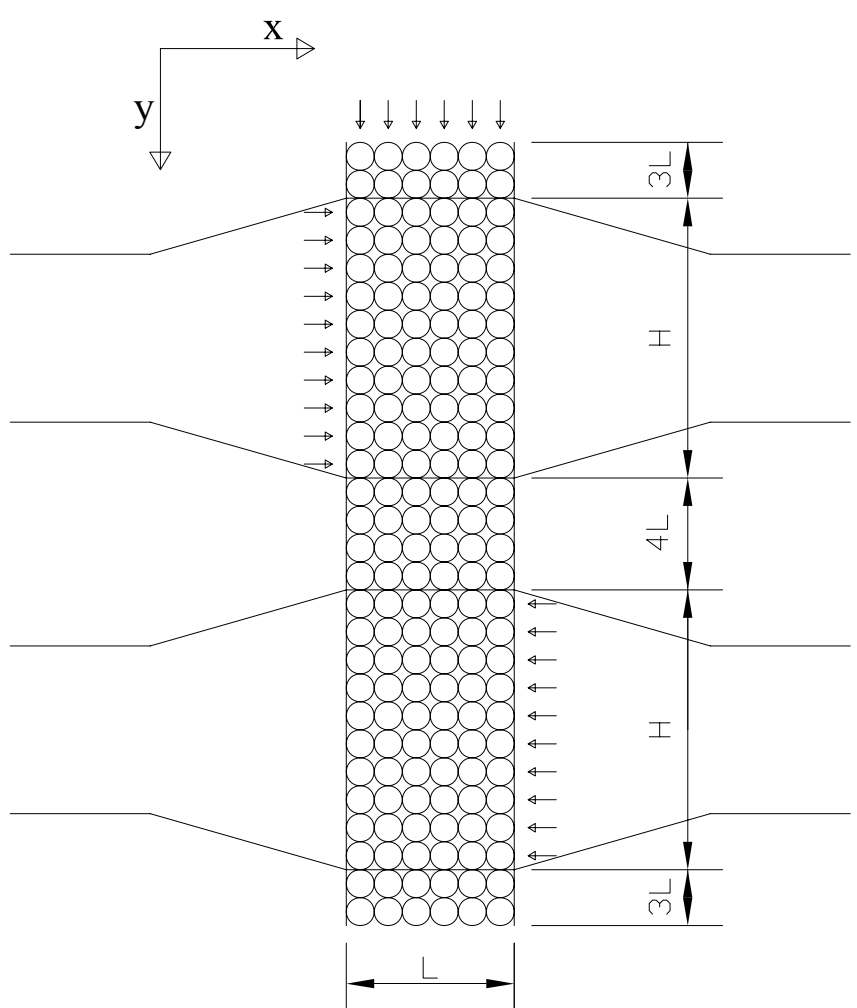

Figure 5. Schematic of the complete regenerator (the graph is out of scale). 


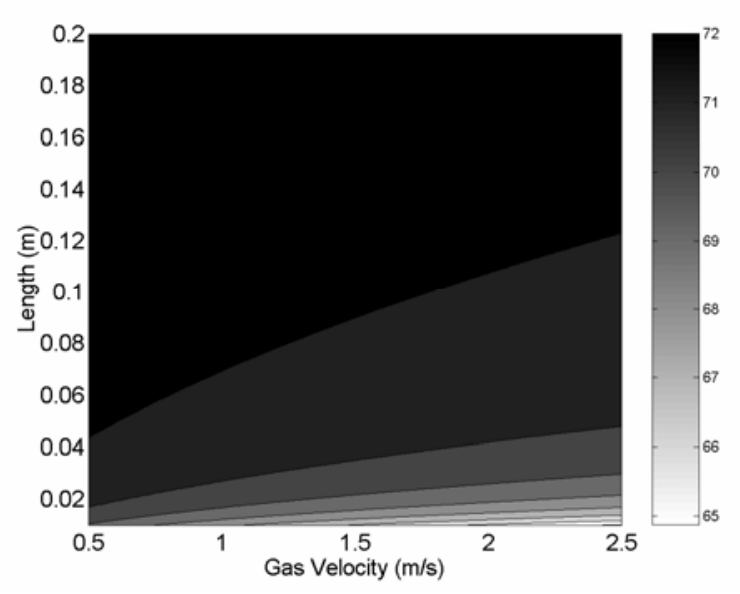

a)

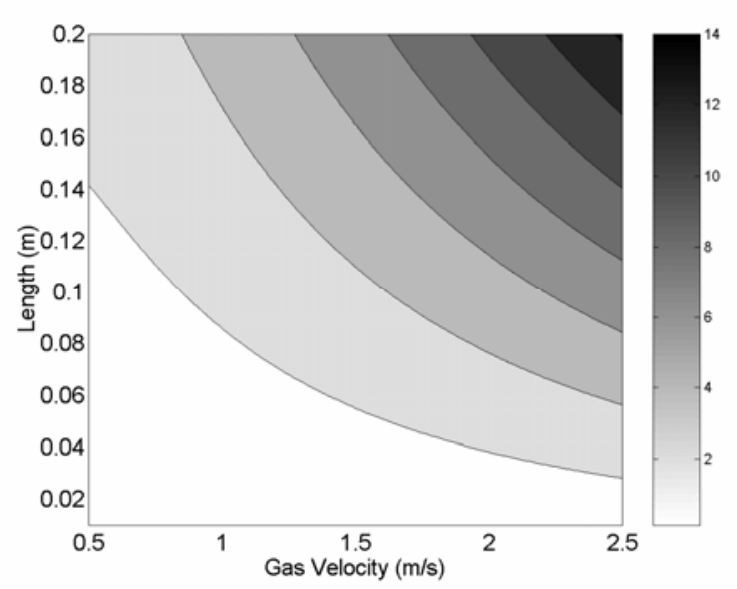

b)

Figure 6: Heat transfer (a) and power consumption (b) per unit of gas mass flow $(\mathrm{kJ} / \mathrm{kg})$

Heat transfer increases with the MBHE length and for decreasing gas velocities. Power consumption increases with both length and gas velocity. While heat transfer increases rapidly at very low values of the length and then stabilizes, power consumption has an almost linear increase with the length. Therefore, an optimal length can be found when the derivatives balance. Values of the length higher than the optimal one produces small increases of heat transfer at the cost of higher increases of power consumption. Similarly, lengths smaller than the optimal one produces important decreases of heat transfer for lower decreases of power consumption.

Figure 7 shows the optimal length calculated as a function of the gas velocity. Once the optimal length, $\mathrm{L}$, is known, the optimal height, $\mathrm{H}$, can be calculated for the square (in a non-dimensional sense) MBHE by rearranging equation ( 8 ).

$$
H=\left(\frac{1-\varepsilon}{\varepsilon} \cdot \frac{\rho_{s}}{\rho_{g}} \cdot \frac{C_{s}}{c_{p g}} \cdot u_{s}\right) \cdot \frac{L}{u_{g}}
$$

Furthermore the optimal width $(\mathrm{T})$ of the MBHE, can be obtained from the definition of the gas mass flow. The width is the only dimensional parameter that depends on the gas mass flow and it is
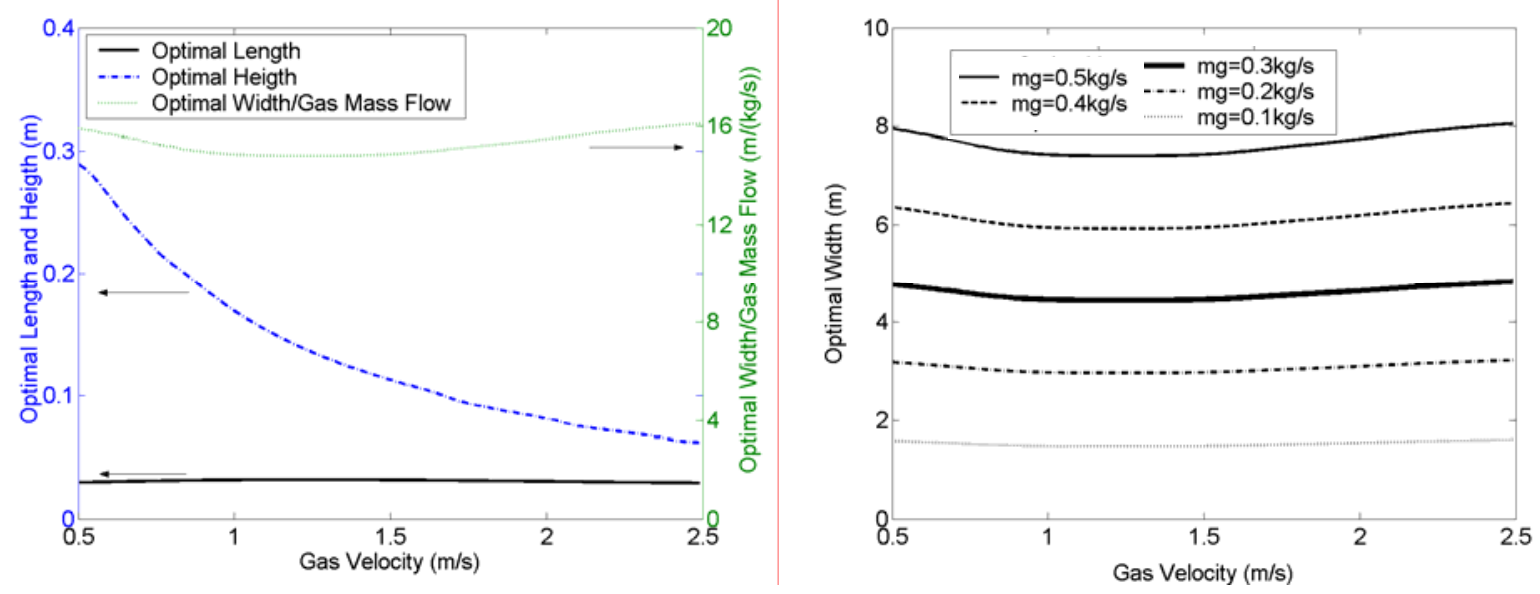

Figure 7: Optimal dimensions of the MBHE: a) Length (L), Height (H) and Width per unit mass flow (T/mf); b) optimal width (T) for different gas mass flows 
given per unit of mass flow by equation (22).

$$
\frac{T}{\dot{m}_{g}}=\frac{1}{\varepsilon \cdot \rho_{g}} \cdot \frac{1}{H \cdot u_{g}}
$$

Figure 7(a) shows the optimal dimensions (L, H, and $\mathrm{T} / \mathrm{m}_{\mathrm{f}}$ ) of the MBHE for a range of gas velocities. $L$ and $T / m_{f}$ are mostly unaffected by the gas velocity, while $H$ varies as $1 / u_{\mathrm{g}}$, as stated in equation (21). Note that $\mathrm{H}$ and $\mathrm{L}$ are not very different, in dimension, while $\mathrm{T}$ may vary depending on the gas mass flow. Figure 7(b) shows the value of this parameter (the optimal width, T) for different values of the gas mass flow around the nominal one $(0.3 \mathrm{~kg} / \mathrm{s})$. For gas mass flows around $0.1 \mathrm{~kg} / \mathrm{s}$ or less, reasonable dimensions of the MBHE are obtained, but for higher values the optimal width is much higher than the optimal length and height, rendering the optimal design unacceptable. Therefore, for large gas mass flows, an optimal regenerator is incompatible with a wellproportioned one and other solutions should be possible.

\section{CONCLUSIONS}

A thermal and exergy analysis is performed over a moving bed heat exchanger (MBHE) and the heat transfer mechanisms and pressure drop inside the bed are analyzed. The heat transfer analysis shows differences in the temperature field when solid conductivity is taken into account, although the mean outlet temperatures do not change notably, especially for high values of $\xi_{\max }$ and $\eta_{\max }$. Consequently, the optimum performance point which minimizes the exergy destroyed during the steady state operation of the MBHE can be computed using the analytical solution neglecting solid conductivity (equations (17) and (18)).

The global optimization of the heat regenerator permits to obtain the optimal dimensions of the device. The length $\mathrm{L}$ and the width $\mathrm{T}$ are not affected notably by the gas velocity. In contrast the optimal height $\mathrm{H}$ decreases as $1 / \mathrm{u}_{\mathrm{g}}$. Finally, an increase in the gas mass flow increases the optimal width $\mathrm{T}$ and an optimal regenerator becomes unfeasible for high gas mass flows.

\section{REFERENCES}

Achenbach, E. [1995], Heat and flow characteristics of packed beds, Experimental Thermal and Fluid Science, Vol. 10, No. 1, pp 17-27

Bejan A. [1996], Entropy generation minimization: The new thermodynamics of finite-size devices and finite-time processes, Journal of Applied Physics, Vol. 79, No 3, pp. 1191-1218

Ergun, S. [1952]. Fluid flow through packed columns, Chemical Engineering Progress, Vol. 48, No. 2, pp 89-94

Henriquez, A. and Macias-Machin, A. [1997], Hot gas filtration using a moving bed heat exchanger-filter (MHEF), Chemical Engineering and Processing, Vol. 36, No. 5, pp 353-361

Hsiau S.S., Smid J., Tsai F.H., Kuo J.T. and Chou C.S. [2004], Placement of flow-corrective elements in a moving granular bed with louvered-walls, Chemical Engineering and Processing, Vol. 43, pp 1037-1045

Hsiau S.S., Smid J., Tsai F.H., Kuo J.T. and Chou C.S. [2001], Velocities in moving granular bed filters, Powder Technology, Vol. 114, pp 205-212 
Hsiau S.S., Smid J., Tsai F.H., Kuo J.T. and Chou C.S. [1999], Velocity profiles of granules in moving bed filters, Chemical Engineering Science, Vol. 54, pp 293-301

Krupiczka, R. [1967], Analysis of thermal conductivity in granular materials, International Chemical Engineering, Vol. 7, No. 1, pp 122-144

Longanbach J.R. [1998], Preparing advanced coal-based power systems for the $21^{\text {st }}$ century at the power systems development facility in Wilsonville, Alabama. In: Proc. $23^{\text {rd }}$ Int. Technical Conf. on Coal Utilization and Fuel Sytems, Clearwater, FL, USA, pp. 69-78

Macías-Machín A., Estevez A., Cuellar J. and Jaraiz E. [1991], Simple design of crossflow movingbed heat exchanger-filter (MHEF), Filtration and Separation, Vol. 29, pp. 155-161

Marb C.M. and Vortmeyer D. [1988], Multiple steady-states of a cross-flow moving bed reactor: theory and experiment, Chemical Engineering Science, Vol. 43, No. 4, pp 811-819

Saastamoinen J.J. [2003], Heat exchange between two coupled fixed beds by fluid flow, International Journal of Heat and Mass Transfer, Vol. 46, No. 15, pp 2727-2735

Smid J., Hsiau S.S., Peng C.Y. and Lee H.T. [2005a], Moving bed filters for hot gas cleanup, Filtration \& Separation, Vol. 42, No. 6, pp 34-37

Smid J., Hsiau S.S., Peng C.Y. and Lee H.T. [2005b], Granular moving bed filters and adsorbers (GM-BF/A) - patent review: 1970-2000, Advanced Powder Technology, Vol. 16, No. 4, pp 301345

Socorro M., Macias-Machin A., Verona J.M. and Santana D. [2006], Hot gas filtration and heat exchange in a packed bed using Lapilli as a granular medium, Industrial \& Engineering Chemistry Research, Vol. 45, No. 23, pp 7957-7966

Soria-Verdugo A., Almendros-Ibáñez J.A., Ruiz-Rivas U., Santana D. [2007], Exergy optimization in a steady moving bed heat exchanger. Proceedings of Interdisciplinary Transport Phenomena $V$ : Fluid, Thermal, Biological, Materials and Space Sciences, 14-19 October, 2007, Bansko, Bulgaria. Paper ITP-07-70. Accepted for publication in "Annals of The New York Academy of Sciences"

Vortmeyer, D. and Schaefer, R.J. [1974], Equivalence of one-phase and 2-phase models for heattransfer processes in packed-beds-one-dimensional theory, Chemical Engineering Science, Vol. 29, No. 2, pp 485-491

Yagi, S. and Kunii, D. [1960], Studies on Heat Transfer Near Wall Surface in Packed Beds, AIChE Journal, Vol. 6, No. 1, pp 97-104

Yagi, S., Kunii, D. and Wakao N. [1960], Studies on axial effective thermal conductivities in packed beds, AIChE Journal, Vol. 6, No. 4, pp 543-546 